\title{
Biaxial Stress Limit for ULDB Film
}

\author{
W.J. Sterling, NASA-GSFC Code 541, Wallops Flight Facility, Virginia \\ J.L. Rand, Winzen Engineering, San Antonio, Texas
}

\begin{abstract}
The current ultra-long duration balloon design applies biaxial stress to the shell film to control latitudinal creep, following Schapery's nonlinear viscoelastic theory. Although a very similar material is used in zero-pressure balloons, the stress state differs significantly. The major implication of biaxial shell loading is that for this material type the minimum in effective stress, which is a measure of the residual forces, occurs at approximately equal loading in the two principal material directions. The shell material capabilities for this project are bound by two criteria: (1) avoid stresses that would cause tertiary creep at relevant temperatures and durations, and (2) limit creep below an extent that would cause a structural instability. Both criteria are investigated via traditional uniaxial creep experiments using thin strips of material bearing steady load. More unique are experiments with pressurized cylinders that may have added axial and/or torsional load to evaluate the biaxial creep response. The Schapery-Rand material constitutive model is tuned via creep experiments with loads in the ULDB design range, while the tertiary creep limit data bound ULDB design.
\end{abstract}

\section{Introduction}

The current ultra-long duration balloon (ULDB, "Pumpkin") design applies stress to the shell film biaxially to control creep in the latitudinal direction. The recent change in design paradigm, from a uniaxial to biaxial stress state basis, arose from a new perspective that the biaxial loading can control strain in both principal surface dimensions as discussed below. The current ULDB project path was thus enabled by a more thorough understanding of the nonlinear viscoelastic properties of the shell film material, linear low-density polyethylene (LLDPE) coextruded to a nominal thickness of 38 microns ( $1.5 \mathrm{mil})$. Although a very similar material is also used in zero-pressure (ZPB) and long-duration balloons (LDB), the different stress state requires a new approach to shell material qualification.

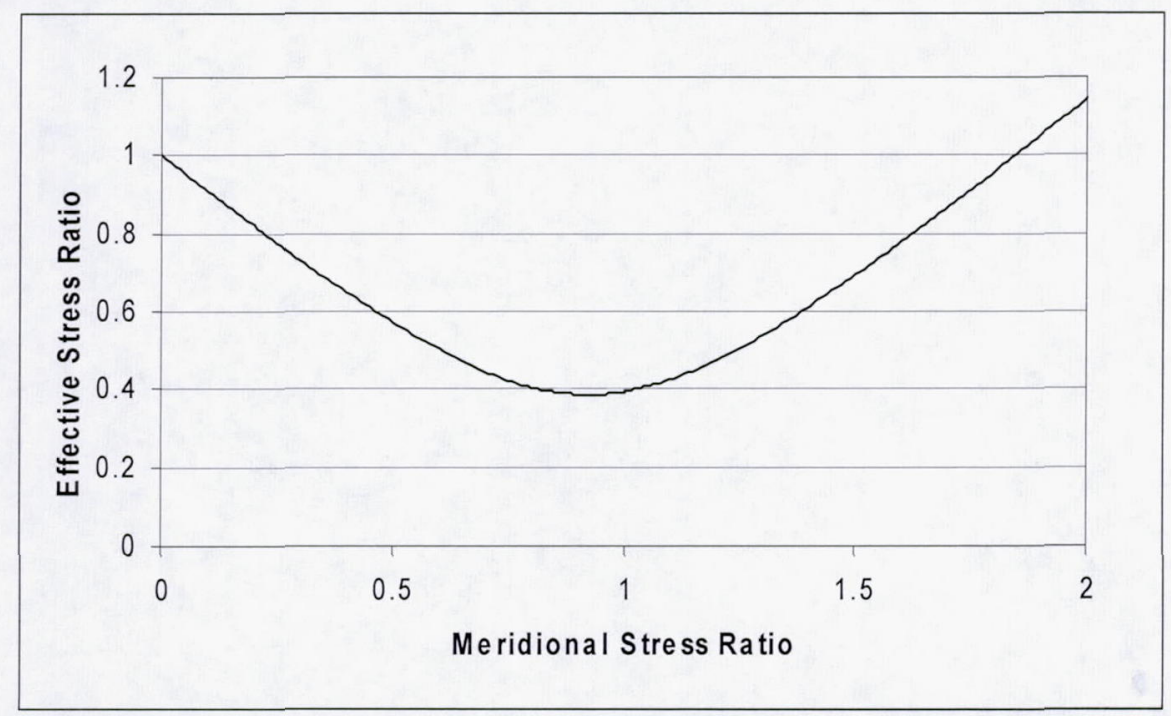

Figure 1. The implications of biaxial loading on film creep. 
The implications of biaxial loading on film creep are displayed in Figure 1 (Rand and Sterling) as the minimum in effective stress at approximately equal loading in the two principal material directions. In this figure, the meridional stress ratio is the ratio of meridional (longitudinal) to hoop (latitudinal) stress, while the effective stress ratio is the ratio of effective stress to hoop stress. Effective stress is a measure of the residual forces that cause biaxial creep (Rand and Sterling.

The principal material coordinates, commonly known as film machine direction (MD) and transverse direction (TD), fortunately correspond directly to the structural coordinates of meridian and hoop, respectively. A design case that applies no load in the balloon film along the meridional direction, such as the ULDB design iteration circa 2002, is seen in Figure 1 to capture none of the compensating benefits of biaxial loading. For a perfectly balanced film, i.e. one with identical properties in the principal material directions, the minimum in effective stress ratio would correspond to a meridional stress ratio of unity.

The approach to determining material capabilities for final design iterations and the development of operational parameters is based on maintaining stress below a level that would cause uncontrolled deformation, or that would cause controlled deformation of the vehicle into an undesired size or shape. The determination of a biaxial loading limit is complicated by the nonlinear viscoelastic nature of the material, which implies that temperature and duration are also key determinants of material capability (Ferry).

One aspect of a loading limit for this application arises due to the stability threshold envelope of the vehicle design. It is required to limit creep below an extent that would cause a structural instability. The creep behavior is represented for ULDB by the nonlinear viscoelastic constitutive model developed by Schapery based on thermodynamic principles. The basic theory and its adaptation for this application by Rand has been thoroughly discussed in prior communications (Schapery, Rand et al, Rand and Sterling). In this paper, we present unique experiments that are used to tune the parameters of the Schapery-Rand constitutive model. The model is being integrated into the vehicle stability analysis software that will be presented elsewhere by those responsible for its development.

The second aspect of the approach to determine the material capabilities for ULDB is to investigate the load limit that avoids stresses that would cause tertiary creep at the temperatures and over the durations relevant to the ULDB project goals. This limit, the tertiary creep onset threshold, is described by a mapping of the region of stable creep as a function of the biaxial stress state expressed in the principal material coordinates. The tertiary onset threshold represents a change in material behavioral regime from one in which a nonlinear viscoelastic constitutive representation such as Schapery-Rand applies, to a regime in which the creep behavior is significantly influenced by a different, more rapid, time effect. Practically, tertiary onset is indicated when creep data viewed as strain versus logarithmic time begin to fall above the stable creep (secondary) trend.

The key parameters for the tertiary creep onset, temperature and duration, were chosen within the ULDB project team. The relevant shell temperatures arose from thermal analyses using the best existing estimates of flight environs and material thermo-optical properties. The relevant duration, 24 hours, arose from the anticipated flight profile for a 100 day flight from Alice Springs, Australia, which would include 3 hot case days of approximately 8 hours duration each. These 3 periods of 8 hours were summed to take the most conservative approach that no 
material recovery between them would occur. The 8 hour estimate was conservative in itself as it assumes the temperature profile to have a square shape.

\section{Experiments}

Traditional uniaxial film strip creep data were collected to guide the biaxial experiment design, and to determine the intercepts of the tertiary creep onset threshold map. Strips were hand cut $50 \mathrm{~mm}$ in width with a $300 \mathrm{~mm}$ gauge length. Loads were applied using cloth bags filled with measured amounts of lead shot. Temperature was maintained at desired levels by an environmental chamber cooled with liquid nitrogen flow that was controlled by home-built devices based on Omega thermocouples and temperature controllers.

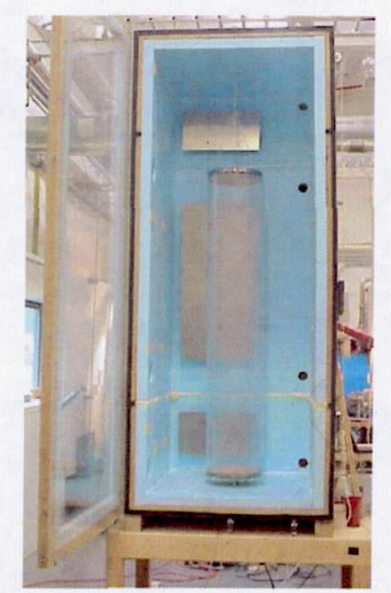

Figure 2. Biaxial characterization apparatus.

Biaxial creep data were collected via experiments using pressurized cylinders to give a 2:1 stress ratio (circumference:axis) and added axial or torsional load to cause other stress states such as 1:1. Cylinders of initial $30 \mathrm{~cm}$ diameter and $100 \mathrm{~cm}$ length (approximate dimensions) were fabricated in each orientation relative to the principal material coordinates. Figure 2 shows one such cylinder assembled inside the (open) environmental chamber that was used to control the temperature in the same manner as the uniaxial experiments. The cylinder end plates were made of $\sim 2 \mathrm{~cm}$ thick plywood and the film was affixed to the plates using hose clamps. Felt padding was used to protect the film from the hose clamp, and rubber padding protected the film from the plywood. Good sealing at the end plates was observed, but was not critical because dry nitrogen pressurization gas was added and vented continuously as needed by a home-built automatic pressure controller. Two valves, one each for filling and venting, were operated by a custom LabVIEW program to control the pressure as desired according to measurement by an MKS Barotron pressure transducer. The loads were applied nominally instantaneously and simultaneously at time zero and data were acquired starting at one minute thereafter. 


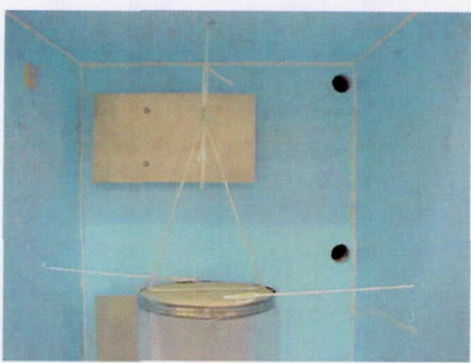

Figure 3. Top plate of pressurized cylinder showing suspension and loading scheme.

The cylinder bottom plate was fixed in place by stand-off legs, while the cylinder top was free to move according to the film response. The top was suspended on a 4-way harness connected to a lever arm with a weight to counterbalance the mass of the top plate (Figure 3 ). Any desired axial load was added to this counterbalance. Torsional loads were applied using hanging weights whose force was directed to measured locations on the top plate by directional pulleys. Strain measurements were made via two Vernier scales, one each along the cylinder axis and hoop, with initial gauge length of $10 \mathrm{~cm}$, as shown in Figure 4.

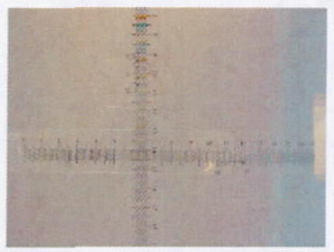

Figure 4. Biaxial strain measurement technique.

Biaxial creep data were acquired to fit the parameters of the Schapery-Rand model at the conditions shown in Table 1 at $283 \mathrm{~K}$, the highest film temperature anticipated on an abnormally hot day. An analogous set of experiments was conducted at $253 \mathrm{~K}$, the highest film temperature anticipated on a normal day, but with stresses reflecting the higher stiffness of the material at the lower temperature. For the determination of biaxial tertiary creep onset threshold, experiments at increasingly higher stresses were conducted and only the determined threshold values are deemed appropriate for presentation (below).

Table 1. Biaxial creep experiment conditions for constitutive parameter fitting at $283 \mathrm{~K}$. 


\begin{tabular}{|cccccccc|}
\hline $\begin{array}{c}\text { Film } \\
\text { Orientation } \\
\text { Axial }\end{array}$ & $\begin{array}{c}\text { Hoop } \\
\text { stress } \\
\mathrm{MPa}\end{array}$ & $\begin{array}{c}\text { Axial } \\
\text { stress } \\
\mathrm{MPa}\end{array}$ & $\begin{array}{c}\text { Shear } \\
\text { stress } \\
\mathrm{MPa}\end{array}$ & $\begin{array}{c}\text { Effective } \\
\text { stress } \\
\mathrm{MPa}\end{array}$ & $\begin{array}{c}\text { Pressure } \\
\mathrm{kPa}\end{array}$ & $\begin{array}{c}\text { Axial } \\
\text { load } \\
\mathrm{kg}\end{array}$ & $\begin{array}{c}\text { Torsion load } \\
\text { each side } \\
\mathrm{kg}\end{array}$ \\
\hline TD & 4.7 & 2.3 & 0.00 & 2.67 & 1.1 & 0.000 & 0.000 \\
TD & 4.7 & 4.7 & 0.00 & 1.83 & 1.1 & 8.430 & 0.000 \\
TD & 4.7 & 4.7 & 1.07 & 2.67 & 1.1 & 8.430 & 2.266 \\
TD & 2.3 & 1.2 & 0.00 & 1.33 & 0.6 & 0.000 & 0.000 \\
TD & 2.3 & 2.3 & 0.00 & 0.92 & 0.6 & 4.215 & 0.000 \\
TD & 2.3 & 2.3 & 0.54 & 1.33 & 0.6 & 4.215 & 1.133 \\
\hline MD & 4.7 & 2.3 & 0.00 & 2.67 & 1.1 & 0.000 & 0.000 \\
MD & 4.7 & 4.7 & 0.00 & 1.83 & 1.1 & 8.430 & 0.000 \\
MD & 4.7 & 4.7 & 1.07 & 2.67 & 1.1 & 8.430 & 2.266 \\
MD & 2.3 & 1.2 & 0.00 & 1.33 & 0.6 & 0.000 & 0.000 \\
MD & 2.3 & 2.3 & 0.00 & 0.92 & 0.6 & 4.215 & 0.000 \\
MD & 2.3 & 2.3 & 0.54 & 1.33 & 0.6 & 4.215 & 1.133 \\
\hline
\end{tabular}

\section{Results}

Figure 5 shows data for two experiments to measure the biaxial creep response at ULDB design-relevant stresses for constitutive model parameter fitting. The experiments shown, which had no axial or torsional load, differ only in cylinder pressure, with data in blue representing twice the pressure of data in red. Open symbols refer to hoop strain, while filled symbols refer to axial strain. Axial strains less than zero represent contraction of the cylinders along the axis due to Poisson's effect (Ferry). Such data have been collected in the twelve loading states shown above in Table 1 covering relevant parameter space in cylinder pressurization, applied axial load, applied torsional load, and cylinder film orientation. The data coarseness that can be observed in Figure 5 is the measurement resolution, plus or minus one step, which is $0.1 \%$ strain or $0.1 \mathrm{~mm}$.

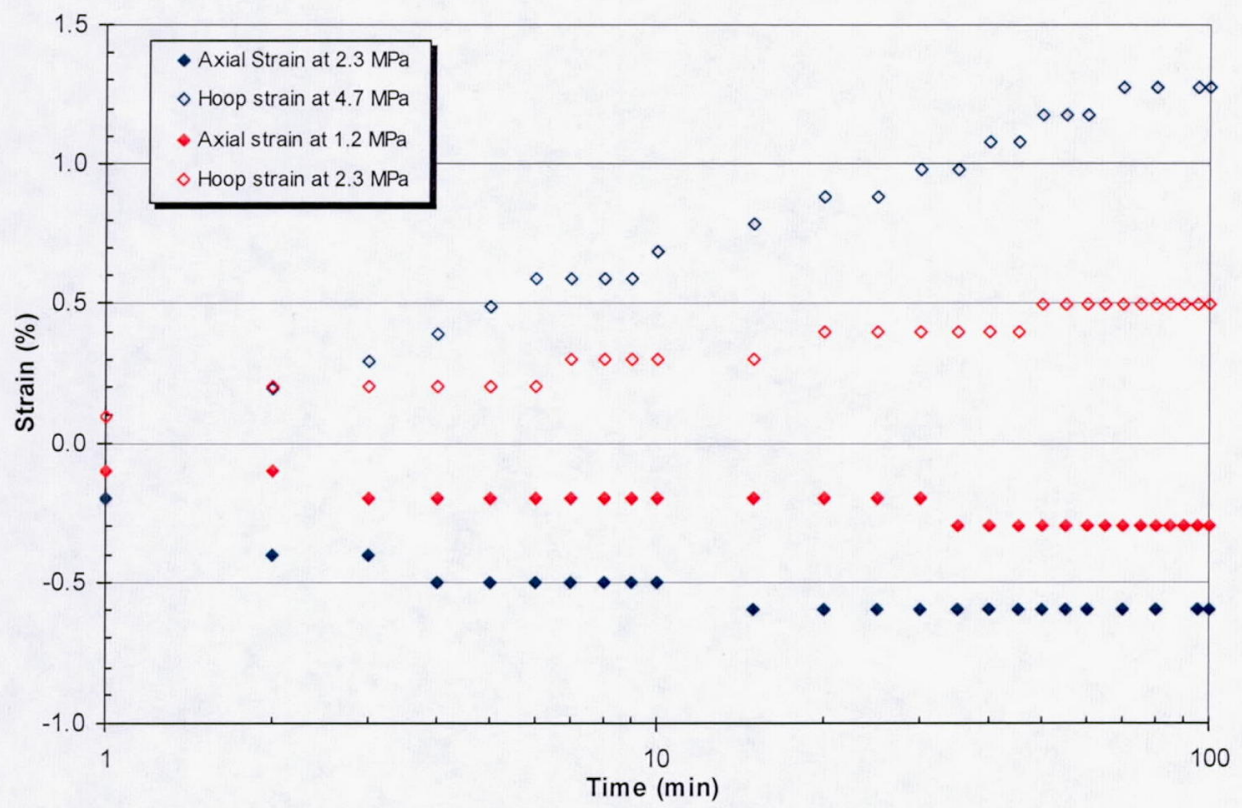

Figure 5. Typical biaxial creep data. 
Uniaxial creep data were collected at numerous temperatures (210K, 243K, 253K, 283K, and $293 \mathrm{~K}$ ) and many stresses in both principal material orientations, plus in an orientation 45 between the principal orientations ("on the bias" in the lexicon of fabrics). Figure 6 shows a large fraction of the data collected at one temperature in the TD orientation. Repeat experiments at $5.5 \mathrm{MPa}$ are shown as evidence of reproducibility. The uniaxial tertiary creep onset threshold for the duration of interest is shown to be $6.5 \mathrm{MPa}$. Trend lines for the lower and higher stresses were constructed to verify the determination, but are omitted from Figure 6.

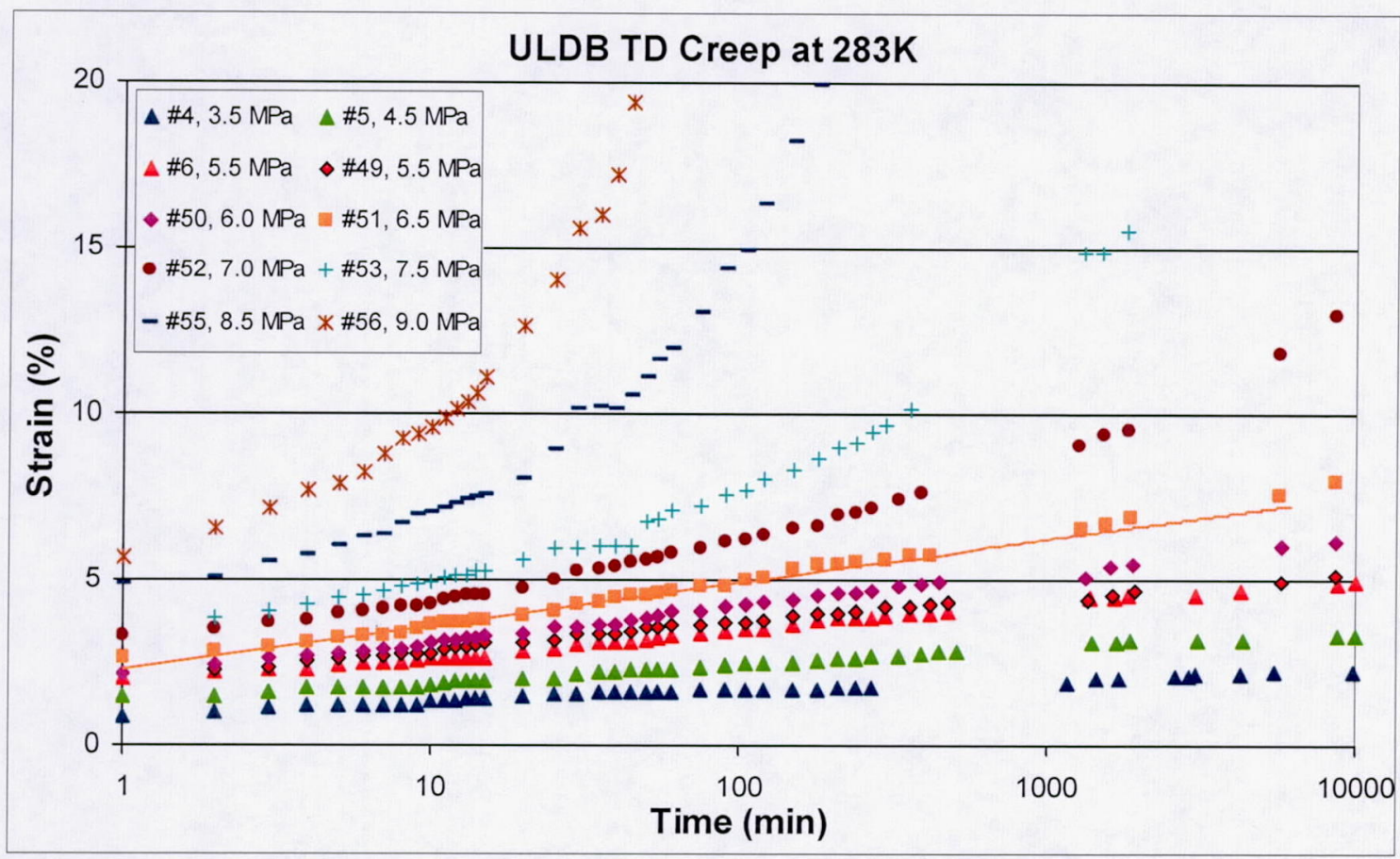

Figure 6. Uniaxial tertiary creep onset determination.

Figure 7 is an example of the biaxial tertiary creep onset determination data. The experiment shown was conducted at $283 \mathrm{~K}$ with $2.3 \mathrm{kPa}$ cylinder pressurization and no axial or torsional load on a cylinder fabricated with the material TD orientation corresponding to the cylinder hoop direction. The data points highlighted in pink were used to determine the stable creep trend lines. Omission of the first two data points is discussed below. The creep in both the hoop and axial cylinder directions entered the tertiary regime at about the same time, approximately 105 minutes, in this experiment. It was more typical that the hoop direction became tertiary prior to the axial direction, but this balance is obviously affected by the biaxial loading conditions. The axial contraction observed in Figure 7 is typical of the results of experiments with gas pressure only, but when axial load was added these strains sometimes became positive. 


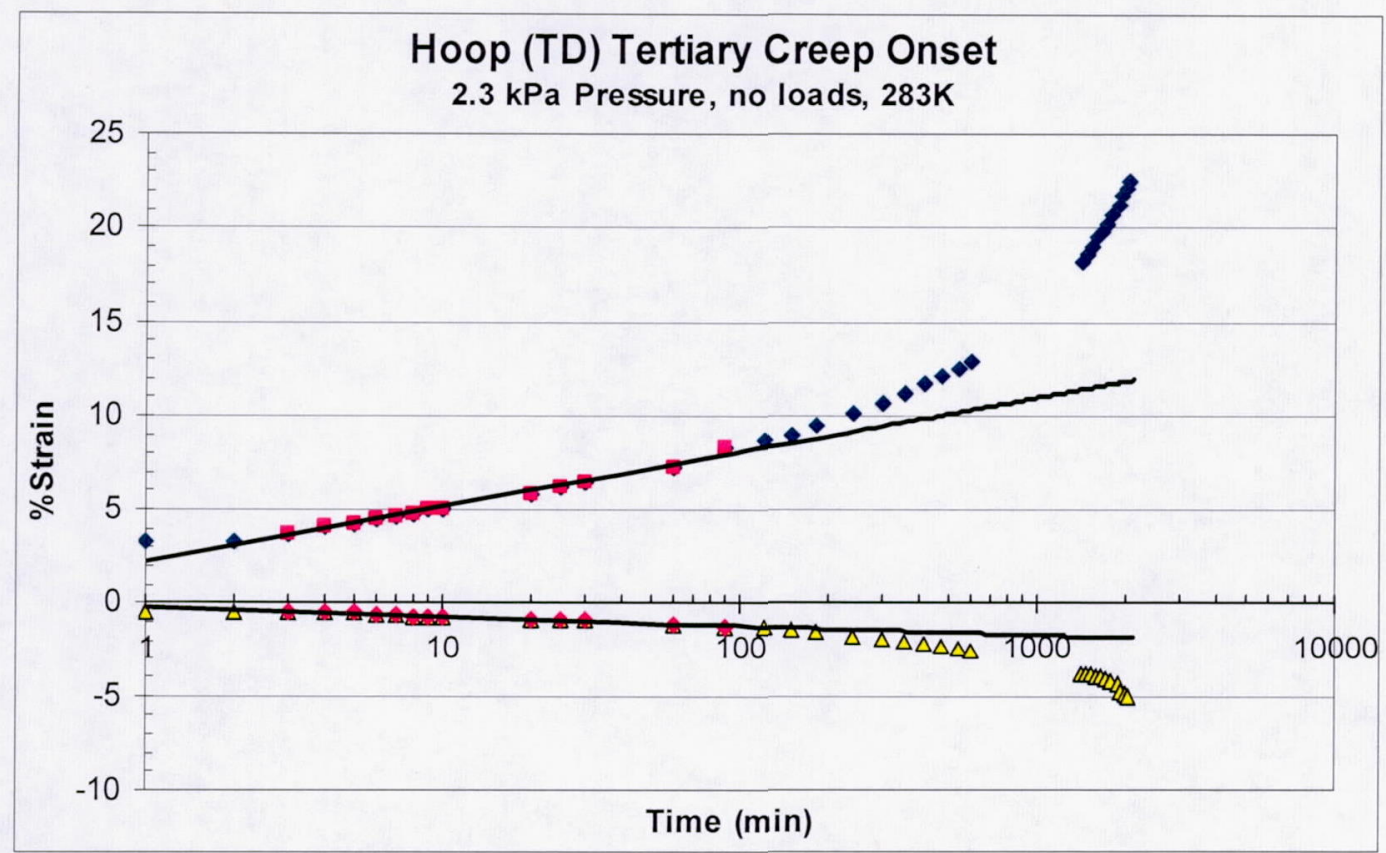

Figure 7. Biaxial tertiary creep determination.

Reported below in Figure 8 and Table 2 are the tertiary creep onset threshold values. Experiments at lower stresses showed stable creep for the defined duration, while at higher stresses the creep was not stable. The data point for the 1:2 stress state (MD:TD) at $210 \mathrm{~K}$ is the threshold value within $1 \mathrm{MPa}$ effective stress. The data point for the 2:1 stress state (TD:MD) at $210 \mathrm{~K}$ was verified to be stable with a repeat experiment, and is supported with stable results at lower stresses, but no tests at higher stress were attempted so the threshold may be higher. Since this value is beyond the uniaxial result, and well beyond the intended material application, no further exploration of the limit was deemed prudent. The point representing the 1:1 stress state at $210 \mathrm{~K}$ is not based on data, but rather a qualitative expectation, and was added to give the curve a more logical shape than would appear without it. 


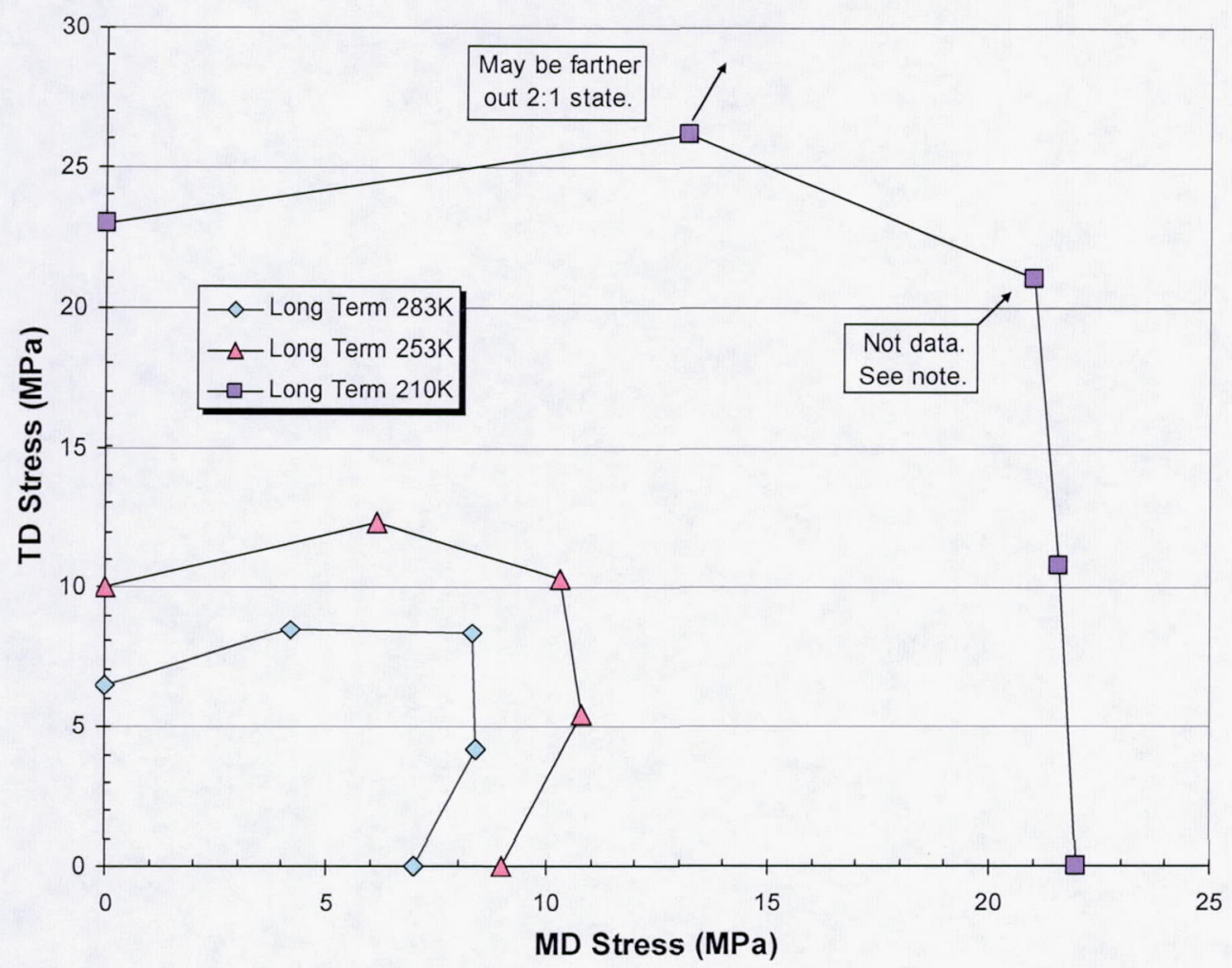

Figure 8. Tertiary creep onset threshold map.

See notes in text.

Table 2. Tertiary creep onset data.

See notes below Figure 1 and in text. "Eff" indicates the calculated effective stress.

\begin{tabular}{|l|c|ccc|ccc|ccc|}
\hline & Cylinder & \multicolumn{3}{|c|}{$210 \mathrm{~K}$} & \multicolumn{3}{c|}{$253 \mathrm{~K}$} & \multicolumn{3}{c|}{ 283K } \\
& Orientation & \multicolumn{2}{|c|}{ Stress (MPa) } & \multicolumn{3}{c|}{ Stress (MPa) } & \multicolumn{3}{c|}{ Stress (MPa) } \\
& (hoop) & MD & TD & Eff & MD & TD & Eff & MD & TD & Eff \\
\hline Uniaxial Tertiary Creep Onset 24 hrs & MD & 22.0 & 0.0 & 22.0 & 9.0 & 0.0 & 9.0 & 7.0 & 0.0 & 7.0 \\
Uniaxial Tertiary Creep Onset 24 hrs & TD & 0.0 & 23.0 & 23.0 & 0.0 & 10.0 & 10.0 & 0.0 & 6.5 & 6.5 \\
Cylinder 2:1 Tertiary Creep Onset 24 hrs & MD & 21.6 & 10.8 & 14.0 & 10.8 & 5.4 & 7.0 & 8.4 & 4.2 & 5.4 \\
Cylinder 2:1 Tertiary Creep Onset 24 hrs & TD & 13.2 & 26.2 & 17.0 & 6.2 & 12.3 & 8.0 & 4.2 & 8.4 & 5.4 \\
Cylinder 1:1 Tertiary Creep Onset 24 hrs & MD & & & & & & & 8.3 & 8.3 & 4.8 \\
Cylinder 1:1 Tertiary Creep Onset 24 hrs & TD & & & & 10.3 & 10.3 & 6.0 & 8.3 & 8.3 & 4.8 \\
\hline
\end{tabular}

ULDB project verification experiments in which the cylinder film stresses exceeded those in the $6 \mathrm{MM} \mathrm{ft}^{3} \mathrm{ULDB}$ design were also conducted. The MD film stress corresponded to the cylinder hoop stress and was $8.8 \mathrm{MPa}$, while the TD film stress corresponded to the cylinder axial stress and was $5.6 \mathrm{MPa}$, so that the calculated effective stress was $5.2 \mathrm{MPa}$. The experiment conducted at $253 \mathrm{~K}$, which is an average daytime high film temperature anticipated in flight, gave the desired result of stable creep for 24 hours. Four experiments at the same loading but $283 \mathrm{~K}$ were conducted and showed good reproducibility, although one was excluded due to a failed heat seal. Hoop strain data for this experiment are shown in Figure 9. The trend for stable creep (triangular points) was determined, and tertiary creep is indicated by the diamond-shaped points to begin sometime after 24 hours of continuous loading, thus meeting the design goal. 


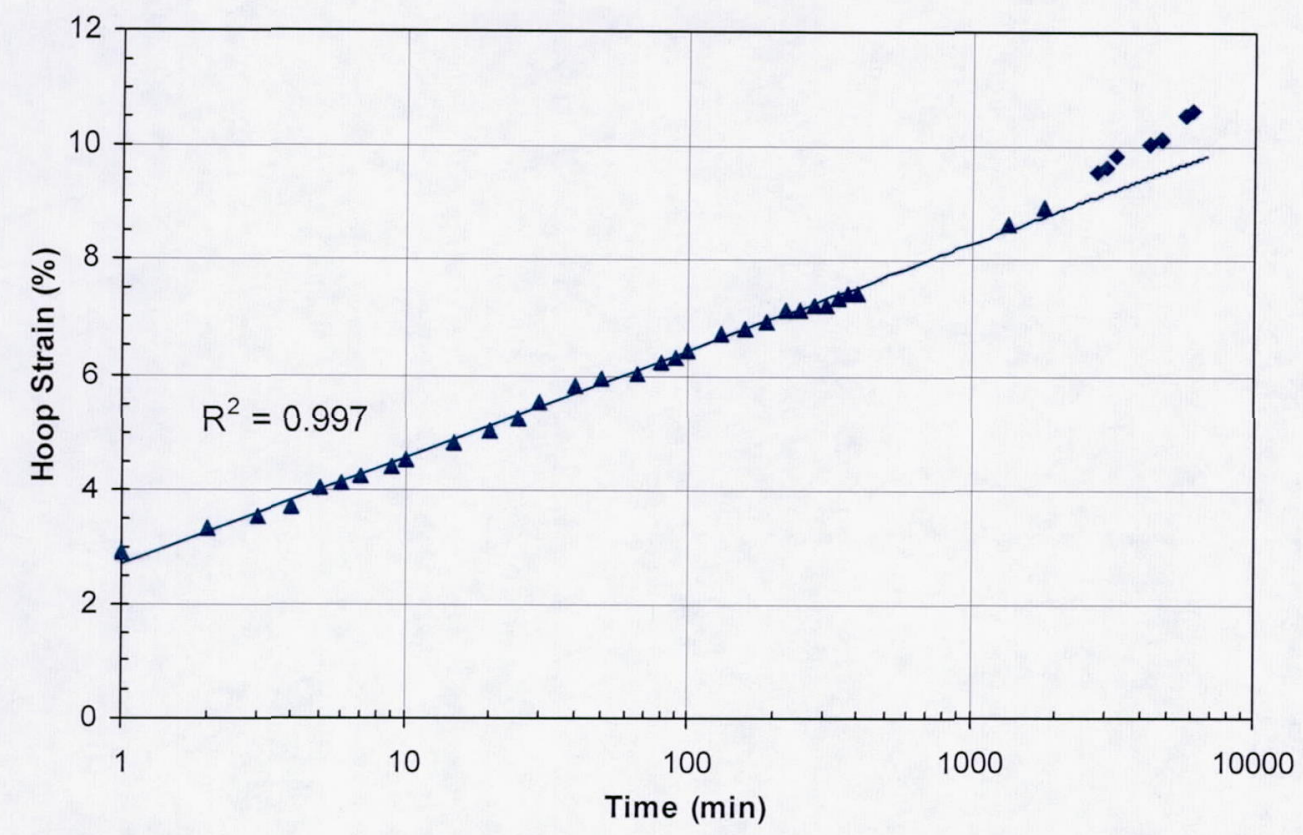

Figure 9. Creep data from experiment applying stresses in excess of the preliminary design for the $6 \mathrm{MM} \mathrm{ft}^{3}$ ULDB. Stable creep duration was at least 24 hours, thus meeting the design goal.

\section{Discussion}

To determine the stable creep trend line for tertiary creep onset time determination, the data between 3 minutes and 105 minutes were used. Due to the observation that the data at 1 and 2 minutes nearly always lay above any potential trend line, it was decided to omit them from the trend prediction. It was also judged that within the 105 minute time, the stable creep trend was clearly established. The determination of creep stability at 24 hours was made by comparing 3 data points around this time to the predicted trend. If the data lay above the trend line, and especially if the strain was moving away from the trend line, the onset of tertiary creep was indicated and the follow-on decision that the loading state was not within the threshold was clear. Due to the loading step size between experiments ( $200 \mathrm{~Pa}$ differential pressure for the 2:1 loading cases and similar for the 1:1 cases), the biaxial stability determination may be as much as $1 \mathrm{MPa}$ conservative for each principal stress.

While working with the data, it was qualitatively observed that the threshold increase for an 8 hour limit, versus the 24 hour limit reported herein, was probably not more than $1 \mathrm{MPa}$. Obviously, any performance data that can be gathered on an upcoming flight will be very valuable in understanding the true degree of conservativeness in this process, and in understanding the true applicability of these measurements.

Note that the data resolution, $0.1 \%$ strain, often appears as the distance separating one point from another or the trend line, giving the appearance in some cases of marked behavior changes when in fact the step is only the data resolution. Given the subjectivity, it seems pertinent to point out that there is no marked effect occurring at the tertiary creep onset. Rather, it is a change in material response regime from one in which a nonlinear viscoelastic constitutive representation such as Schapery-Rand applies, to a regime in which the creep behavior is significantly influenced by a different, more rapid, time effect and plasticity may contribute.

It is encouraging to note that the shape of the biaxial tertiary creep onset threshold is supported by theory and experiments outside our efforts. Gol'dman, in his exhaustive monograph 
on polymer deformation, provides both theory and experiments with a variety of materials that confirm the Schapery development and the shape of the tertiary creep onset threshold map. Further, as verification of self-consistency, the Schapery-Rand theory predicts the tertiary creep onset map might realistically be represented by an effective stress contour for each temperature. When plotting the effective stress using the constitutive parameters determined for the balloon film, the general shape predicted by Figure 8 is confirmed. However, rather than fitting the constitutive parameters with the tertiary creep onset threshold data via the effective stress, it is more prudent to acknowledge the effective stress as a computation internal to the constitutive model and to therefore fit the more directly relevant creep data instead.

The Schapery-Rand constitutive model has only very recently been integrated into the ULDB structural analysis software. This software also has the capability to model deforming cylinders, and will therefore be employed to tune the material constitutive parameters according to the data reported herein. Presently, the model parameters have been adjusted according to the ULDB film uniaxial data and extensive ZPB data as reported previously (Rand and Sterling). In preliminary work along these lines, it appears that having data with finer resolution might be beneficial, and therefore a subset of these experiments might be repeated using a photogrammetric technique (Blandino et al.) to measure strain instead of the technique reported herein.

\section{Conclusions}

The determination of a ULDB film loading threshold in terms of the unconstrained pressurized cylinder stress state has been completed insofar as can be accomplished given present laboratory resources. The data at $210 \mathrm{~K}$ were significantly more difficult to obtain and are thereby less reliable than those at $253 \mathrm{~K}$ and $283 \mathrm{~K}$. The loading threshold at $210 \mathrm{~K}$ is significantly greater than that at the warmer temperatures, and is beyond the intended ULDB stresses.

The creep experiments and material constitutive model discussed in this work are being used to set design and operational limits for ULDB. The biaxial data presented are difficult to acquire and are of a nearly unique variety. Obviously, a safety factor will be applied to the limits determined by the material capability assessment to arrive at the design limits. Balloon flight operations are likely to remain on the safe side of the design limits. Therefore, these experiments and the material constitutive model are being used as part of a conservative approach to materials qualification for ULDB.

\section{Acknowledgments}

The authors thank the numerous people who contributed to the design, construction, and operation of the biaxial properties measurement appparatus, including Bruno Munoz, Chris Hoffman, Jon Davis, Bob Ray, Joe Ruffing, Gabe Garde, Lana Budd, Greg Parks, Ted Schultz, and Roy Tolbert. This work was suppported by NASA's Balloon Program Office, which is based at Goddard Space Flight Center's Wallops Flight Facility.

\section{References}

Blandino, J.R., W.J. Sterling, F. Baginski, E. Steadman, J.T. Black, and R.S. Pappa, "Optical Strain Measurement of an Inflated Cylinder using Photogrammetry with Application to Scientific Balloons," AIAA Paper No. 2004-1500, $5^{\text {th }}$ Gossamer Spacecraft Forum, Palm Springs, CA, 2004. 
Ferry, J.D., Viscoelastic Properties of Polymers, John Wiley \& Sons., New York, 1980.

Gol'dman, A.Ya., Prediction of the Deformation Properties of Polymeric and Composite Materials, particularly section 3.3, translated and edited by M. Shelef and R.A. Dickie, ACS Professional Reference Book, American Chemical Society, Washington, D.C., 1994.

Rand, J.L., D.A. Grant, and T. Strganac, "The Nonlinear Biaxial Characterization of Balloon Film," AIAA Paper No. 96-0574, AIAA $34^{\text {th }}$ Aerospace Sciences Meeting \& Exhibit, Reno, NV, 1996.

Rand, J.L., and W.J. Sterling, "A Constitutive Equation for Stratospheric Balloon Materials," Adv. Space Research (in press), and $35^{\text {th }}$ COSPAR Scientific Assembly, Paris, 2004.

Schapery, R.A., "Nonlinear Viscoelastic and Viscoplastic Constitutive Equations Based on Thermodynamics," Mechanics of Time-Dependent Materials, v.1, p. 209-240, 1997.

Schapery, R.A., "On the Characterization of Nonlinear Viscoelastic Materials," Polymer Engineering and Science, vol. 9, no. 4, p. 295-310, 1969. 